\title{
A CARLESON ESTIMATE FOR THE COMPLEX MONGE-AMPERE OPERATOR
}

\author{
MATS ANDERSSON
}

Let $D$ be a pseudoconvex domain in $\mathbb{C}^{n}$ that admits a plurisubharmonic defining function $\rho$ of class $C^{2}$. We prove that if $u_{1}, \ldots, u_{r}$ are bounded plurisubharmonic functions in $D$ and $\omega=d d^{c} \log 1 /(-\rho)$, then $(-\rho)^{n} d d^{c} u_{1} \wedge \ldots \wedge d d^{c} u_{r} \wedge \omega^{n-r} /(n-r)$ ! is a Carleson measure. This is a global variant of the ChernLevine-Nirenberg inequality.

\section{Introduction.}

Let $u_{n}, \ldots, u_{n}$ be bounded plurisubharmonic functions in some domain $\Omega$ in $\mathbb{C}^{n}$. It was proved in [2] and [3] that the Monge-Ampere expression $d d^{c} u_{1} \wedge \ldots \wedge d d^{c} u_{n}$ defines a positive measure (defined via an appropriate regularization), and the Chern-Levine-Nirenberg inequality, see [6], states that for each $K \subset \subset \Omega$ there is a $C=C(K, \Omega)$ such that

$$
\int_{K} d d^{c} u_{1} \wedge \ldots \wedge d d^{c} u_{n} \leq C \sup _{\Omega}\left|u_{1}\right| \ldots \sup _{\Omega}\left|u_{n}\right|
$$

It follows from the proof that one can replace $\sup _{\Omega}\left|u_{j}\right|$ by $\int_{\Omega}\left|u_{j}\right|$ in this estimate for one of the functions $u_{i}$.

The purpose of this note is to prove a global version of this inequality. Let $D$ be a pseudoconvex domain in $\mathbb{C}^{n}$ that admits a plurisubharmonic defining function $\rho$ of class $C^{2}$. Let $d^{c}=(i / 2)(\bar{\partial}-\partial), \omega=d d^{c} \log (1 /-\rho), \beta=d d^{c} \rho$, $\omega_{k}=\omega^{k} / k$ ! and $\beta_{k}=\beta^{k} / k$ !. Moreover, let $H^{1}$ be the space of holomorphic functions in $\Omega$ such that

$$
\|f\|_{H^{1}}=\sup _{\epsilon_{0}>\epsilon>0} \int_{\rho=-\epsilon}|f| d \sigma
$$

is finite, where $d \sigma$ is the surface measure $d \sigma=d^{c} \rho \wedge \beta_{n-1}$. We say that a positive measure $\tau$ in $D$ is a Carleson measure if the estimate

$$
\int_{D}|\phi| d \tau \leq C\|\phi\|_{H^{1}}, \quad \phi \in H^{1}
$$


holds.

Theorem 1.1. Let $u_{1}, \ldots, u_{r}$ be positive plurisubharmonic functions in $D($ and $r \geq 1)$. Then

$$
d \tau=(-\rho)^{n} d d^{c} u_{1} \wedge \ldots \wedge d d^{c} u_{r} \wedge \omega_{n-r}
$$

is a Carleson measure with norm

$$
\leq c_{r, n} e \sup u_{1} \ldots \sup u_{r}
$$

where $c_{n, r}=(r-1) ! n ! /(n-r) !$.

A few comments are in order.

If $u_{j}$ are bounded and plurisubharmonic it follows that $d \tau$ is a Carleson measure with norm $\leq c_{n, r} e 2^{r}\left\|u_{1}\right\|_{L^{\infty}} \ldots\left\|u_{k}\right\|_{L^{\infty}}$.

Clearly Theorem 1.1 implies the Chern-Levine-Nirenberg inequality. The variant with $L^{1}$ norm of one of the $u_{j}$ follows from Proposition 2.1 below and its proof.

Theorem 1.1 is sharp. In fact, restricted to one variable it says that $-\rho \Delta u$ is a Carleson measure in $D$ if $u$ is a bounded subharmonic function. This fact was noted in [4] and it is also shown in [4] that any Carleson measure in the unit disk that satisfies a small regularity assumption is given by such an expression, simply by taking $\phi$ as the Green potential of $\mu /(1-|z|)$. If $k=1$ and $D$ is the ball, then $\omega$ is the Bergman metric form and Theorem 1.1 then says that $\left(1-|z|^{2}\right)^{-1} \Delta u$ is a Carleson measure, where $\Delta$ is the Bergman Laplacian (the invariant Laplacian) $\Delta=\left(1-|z|^{2}\right)\left(\sum \partial^{2} / \partial z_{j} \partial \bar{z}_{j}-\sum z_{j} \bar{z}_{k} \partial / \partial z_{j} \partial \bar{z}_{k}\right)$. This was also stated in [4]. Even in this case the converse holds for reasonable $\mu$, see [5]. (A similar statement holds for the Euclidean Laplacian $\Delta_{E}$; that $\left(1-|z|^{2}\right) \Delta_{E} u$ is a Carleson measure. Just let $u_{2}, \ldots, u_{n}$ be $|z|^{2}$ or $\rho$ or use (2.2) and then proceed as in the proof of Theorem 1.1. However, by the same argument as in [4] it follows that $\left(1-|z|^{2}\right) \Delta_{E} u$ even is a $\mathbb{R}^{2 n}$-Carleson measure.)

In a forthcoming paper, [1], Theorem 1.1 will be used to obtain new estimates for the so-called $H^{p}$-corona theorem in strictly pseudoconvex domains in $\mathbb{C}^{n}$.

\section{Proof.}

The proof of Theorem 1.1 is based on the following proposition. 
Proposition 2.1. Let $u_{1}, \ldots, u_{r}$ be positive plurisubharmonic functions in $C^{\infty}(\bar{D})$. Then

$$
\int_{D}(-\rho)^{n} d d^{c} u_{1} \wedge \ldots \wedge d d^{c} u_{r} \wedge \omega_{n-r} \leq c_{n, r} \sup u_{1} \ldots \sup u_{r-1} \int_{\partial D} u_{r} d \sigma
$$

where $c_{n, r}=(r-1) ! n ! /(n-r) !$.

This proposition can be considered as a global variant of the Chern-LevineNirenberg estimate, [6]. From Proposition 2.1 we can easily derive our main theorem.

Proof of Theorem 1.1. To begin with we assume that all the functions $u_{j}$ as well as $\phi$ are smooth up to the boundary. Notice that

$$
e^{v} d d^{c} v \leq d d^{c} e^{v}
$$

for any real function $v$. Therefore we get for any $t>0$,

$$
t d d^{c} u|\phi| \leq e^{t u+\log |\phi|} d d^{c} t u \leq e^{t u+\log |\phi|} d d^{c}(t u+\log |\phi|) \leq d d^{c} e^{t u+\log |\phi|} .
$$

If we just replace $u_{r}$ in Proposition 2.1 by $\exp \left(u_{r}+\log |\phi|\right)$ we get that

$$
\int_{D}|\phi| d \tau \leq c_{n, r} \sup u_{1} \ldots \sup u_{r-1} \frac{1}{t} \sup e^{t u_{r}} \int_{\partial D}|\phi| d \sigma .
$$

Taking $t=1 / \sup u_{r}$ we get the desired estimate for smooth functions.

The general case is then obtained by applying this result to smaller domains and a regularization procedure. Let $u_{1}, \ldots, u_{r}$ be arbitrary positive plurisubharmonic functions in $D$ and let $D_{\epsilon}=\{\rho<-\epsilon\}$. By a standard regularization there are smooth plurisubharmonic $u_{j}^{\delta}$ that decrease to $u_{j}$ in a neighborhood of $\overline{D_{\epsilon}}$ when $\delta \rightarrow 0$. According to Theorem 2.1 in [3],

$$
\tau_{\delta}=d d^{c} u_{1}^{\delta} \wedge \ldots d d^{c} u_{r}^{\delta} \wedge \omega_{n-r} \rightarrow d d^{c} u_{1} \wedge \ldots d d^{c} u_{r} \wedge \omega_{n-r}=\tau
$$

weakly as measures in this neighborhood. From the smooth case (applied to $\left.D_{\epsilon}\right)$ we now have

$$
\int_{D_{\epsilon}}(-\rho-\epsilon)^{n} \tau_{\delta}|\phi| \leq c_{n, r} \int_{\partial D_{\epsilon}}|\psi| d \sigma \leq c_{n, r}\|\phi\|_{H^{1}} .
$$

Hence,

$$
\int_{D_{\epsilon}}(-\rho-\epsilon)^{n} \tau|\psi| \leq \liminf _{\delta} \int_{D_{\epsilon}}(-\rho-\epsilon)^{n} \tau_{\delta}|\psi| \leq c_{n, r}\|\psi\|_{H^{1}}
$$


and by monotone convergence it finally follows that

$$
\int_{D}(-\rho)^{n} \tau|\psi| \leq c_{n, r}\|\psi\|_{H^{1}}
$$

Proof of Proposition 2.1. As expected the proof consists in a number of integrations by parts. The crucial point is to keep check of all signs so that negative terms can be discarded.

To begin with we claim that if $a$ is a closed (smooth) $(r, r)$-form, then

$$
\int_{D}(-\rho)^{n} \omega^{n-r} \wedge a=\frac{n}{r} \int_{D}(-\rho)^{r} \beta^{n-r} \wedge a .
$$

In fact,

$$
\int_{D}(-\rho)^{n} \omega^{n-r} \wedge a=\int_{D}(-\rho)^{r} \wedge \beta^{n-r} \wedge a-\frac{(n-r)}{r} \int_{D} d(-\rho)^{r} \wedge d^{c} \rho \wedge \beta^{n-r-1} \wedge a
$$

and an integration by parts in the last integral then yields (2.2).

Lemma 2.2. If $a$ is a positive closed $(r-1, r-1)$-form, $u$ is positive and plurisubharmonic and $\alpha>2$, then

$$
\int_{D}(-\rho)^{\alpha} d d^{c} u \wedge a \leq \sup u \alpha \int_{D}(-\rho)^{\alpha-1} a \wedge \beta .
$$

Proof of Lemma 2.2. First notice that

$$
\begin{aligned}
d d^{c}(-\rho)^{\alpha} & =\alpha(\alpha-1)(-\rho)^{\alpha-2} d \rho \wedge d^{c} \rho-\alpha(-\rho)^{\alpha-1} d d^{c} \rho \\
& \leq \alpha(\alpha-1)(-\rho)^{\alpha-2} d \rho \wedge d^{c} \rho .
\end{aligned}
$$

Hence, since $d \rho \wedge d^{c} \rho$ is a positive form,

$$
\begin{aligned}
\int_{D}(-\rho)^{\alpha} d d^{c} u \wedge a & =\int_{D} u d d^{c}(-\rho)^{\alpha} \wedge a \\
& \leq \sup u \int_{D} \alpha(\alpha-1)(-\rho)^{\alpha-2} d \rho \wedge d^{c} \rho \wedge a .
\end{aligned}
$$

However, since $a$ is closed we can replace $\alpha(\alpha-1)(-\rho)^{\alpha-2} d \rho \wedge d^{c} \rho$ by $\alpha(-\rho)^{\alpha-1} d d^{c} \rho$ on the right hand side of (2.5), since, in view of the equality in (2.4), the difference is a closed form that vanishes on the boundary. This proves the lemma. 
It is now easy to conclude the proof of Proposition 2.1. By repeated use of the lemma we get, for $\alpha>r$, that

$$
\begin{aligned}
& \int_{D}(-\rho)^{\alpha} d d^{c} u_{1} \wedge \ldots d d^{c} u_{r} \wedge \beta^{n-r} \\
& \leq \alpha \ldots(\alpha-r+2) \sup u_{1} \ldots \sup u_{r-1} \int_{D}(-\rho)^{\alpha-r+1} d d^{c} u_{r} \wedge \beta^{n-1} .
\end{aligned}
$$

Moving $d d^{c}$ from $u_{r}$ to $(-\rho)^{\alpha-r+1}$ in the last integral and applying the inequality in (2.4) we get

$$
\int_{D}(-\rho)^{\alpha} d d^{c} u_{1} \wedge \ldots d d^{c} u_{r} \wedge \beta^{n-r} \leq \alpha \ldots(\alpha-r) \int_{D}(-\rho)^{\alpha-r-1} u_{r} d \rho \wedge d^{c} \rho \wedge \beta^{n-1} .
$$

When $\alpha \rightarrow r$ this becomes

$$
\int_{D}(-\rho)^{r} d d^{c} u_{1} \wedge \ldots d d^{c} u_{r} \wedge \beta^{n-r} \leq r !(n-1) ! \int_{\partial D} u_{r} d \sigma
$$

Combining with (2.2) we finally get (2.1).

\section{References}

[1] M. Andersson and H. Carlsson, Estimates of solutions of the $H^{p}$ and BMOA corona problem, preprint, Göteborg, 1998.

[2] E. Bedford and B.A. Taylor, The Dirichlet problem for a complex Monge-Ampere equation, Invent. Math., 37 (1976), 1-37.

[3] , A new capacity for plurisubharmonic functions, Acta Math., 149 (1982), $1-40$.

[4] B. Berndtsson, $\bar{\partial}_{b}$ and Carleson type inequalities, Complex Analysis II, Univ. of Maryland, 1985-86, Springer Lecture Notes, 1276 (1987), 42-54.

[5] M. Borke, Carleson measures in the unit ball of $\mathbb{C}^{n}$, preprint, Göteborg, 1991.

[6] S.S. Chern, H.I. Levine and L. Nirenberg, Intrinsic norms on a complex manifold, Global Analysis (Papers in honor of K. Kodaira), Univ. of Tokyo Press, Tokyo, (1969), 119-139.

Received November 7, 1996 and revised February 12, 1997.

Chalmers University of TeChNology

S-412 96 GÖTEBORG, SwEDEN

E-mail address: matsa@math.chalmers.se 